\title{
EDITORIAL
}

\section{'A rose by any other name would smell as sweet', or would it?}

Bone Marrow Transplantation (2013) 48, 881-883; doi:10.1038/ bmt.2012.275; published online 4 February 2013

Was Shakespeare right? (Romeo and Juliet ca.1594) Does it really not matter what we call a flower or in our case some of our transplant procedures and outcomes? In fact, considerable data from experimental psychology (discussed in a prior report) in Bone Marrow Transplantation indicate that Shakespeare was wrong; the descriptor we use for flowers, wine or other objects strongly influences our perception of these objects. ${ }^{1}$

Dictionary.com, the 21st Century replacement for the OED, defines jargon as the language peculiar to a particular trade, profession or group. However, it offers three additional definitions which are enlightening: (1) unintelligible or meaningless talk or writing (gibberish); (2) any talk or writing that one does not understand and (3) language characterized by uncommon or pretentious vocabulary and convoluted syntax, and is often vague in meaning.

The value of jargon is to facilitate communication within a field. However, in many instances use of jargon inhibits communication with outsiders. But there are other, more serious dangers to using jargon. Miscommunication and imprecision are high on this list. This may be acceptable in some fields where perceptions are the key, like listening to music, but what happens when jargon creeps into science? The result can be destructive.

Hematopoietic cell transplant experts are highly specialized. Consequently, it is not surprising that the transplant field has developed a jargon to facilitate communication and describe what its members do. Terms such as SCT, myelo-ablative transplant, PBSC transplant, autologous GvHD (auto-GvHD) and many others are widely used by transplant experts. And although use of these terms facilitates communication amongst transplant experts, several terms are imprecise at best, and incorrect and misleading at worst. This is especially troublesome in science where accuracy and precision are needed. We review several widely used terms we think should be abandoned because they are inaccurate, incorrect or are likely to be misunderstood by persons not expert in transplants.

Stem cell transplant. Stem cell is a term widely used by biologists; this year's Nobel Prize in Physiology or Medicine was given for research in this field. But what are stem cells really and what do we mean when we use this term?

In developmental biology, a stem cell is a cell with an unlimited self-renewal capacity able to differentiate into terminally differentiated cells of a biological system. There are two main categories of stem cells: (1) embryonic stem cells (ES), and (2) somatic or adult stem cells. ES cells, which develop in the embryonic inner cell mass, are pluripotent and can generate all cell lineages of the adult organism. Somatic or adult stem cells also develop in the embryo and retain self-renewal capacity throughout life, but can develop into some but not all cell lineages of the adult organism. Induced pluripotent stem cells are a type of pluripotent stem cell artificially derived from a non-pluripotent cell-typically an adult somatic cell-by inducing expression of specific genes. Induced pluripotent stem cells are similar to ES cells in many aspects, but the full extent of their relationship to natural pluripotent stem cells is unknown.
The definition of stem cell varies based on the field of study (regenerative medicine, aging, gene therapy and so on), the organism being studied (Drosophila sp., mice, humans and so on), persistence through life and other variables. For example, in some organisms, such as Planarians sp., pluripotent cells are maintained throughout life, whereas mouse and human ES cells, which are also pluripotent, are present only transiently during embryonic development. $^{2}$

Another term sometimes confused with stem cell is progenitor cell. Progenitor cells are usually defined as immature cells that can differentiate into specific cell types. Progenitor cells have much less proliferative potential than stem cells. We also need to consider the term precursor cell. Precursor cells are post-mitotic, partially differentiated cells that are usually uni- rather than toti-, pluri- or multipotent. However, neither progenitor cells nor precursor cells are typically categorized as stem cells by biologists.

These somewhat arbitrary definitions of cell types including stem cells are summarized in the Table 1. Misuse of these terms can result in confusion regarding which therapeutic effects might result from the manipulation or transplantation of these cells. Given these considerations it is appropriate to ask whether the widely used term in our field, stem cell transplants, accurately describes what happens when we do a blood cell or bone marrow transplant in humans.

Most hematologists believe that human BM and blood (under special circumstances) contain hematopoietic stem cells (HSCs). However, HSCs lack critical features of stem cells as defined in the Table 1, and are more accurately defined as a subset of progenitor cells derived from embryonic mesoderm. Biological features of true HSCs should include: (1) multipotency and asymmetrical cell divisions that can give rise to different cell types; (2) persistence in a quiescent state and a slow rate of self renewal; (3) ability to remain in an undifferentiated state in specific microenvironment sites called stem cell niches; (4) ability to restore BM function in lethally irradiated animals including rodents and primates and (5) ability to differentiate into diverse hematopoietic lineages including RBCs, myeloid and lymphoid cells and megakaryocytes.

Based on these considerations we suggest that using stem cell transplants to describe what we do in humans is inaccurate and should be abandoned. We are not Luddites and realize that this is a popular jargon that can increase the likelihoods of funding and publication. However, its use is inaccurate, assumes facts for which we have no evidence and is likely to mislead our colleagues and worse, ourselves. In reality, use of artificially genetically marked BM cells in mice and humans strongly suggests we are not transplanting stem cells. Hematopoietic cell transplants or BM and blood cell transplants are better, more accurate terms.

Peripheral blood cell transplants. This term poses a different type of problem. The peripheral modifier implies there is some other type of blood which might be mistaken for what we are transplanting. Where this term came from is unclear. Is there a central blood that needs to be distinguished from peripheral blood? Doesn't the blood we might sample from a vein next return to a more central location such as the heart? Years ago (but in the lifetime of the authors), microbiologists inoculated mice and guinea pigs with pathogens such as trypanosomes, killed the animals and sampled blood from their heart, which was termed heart's blood. ${ }^{3,4}$ There are even several books with heart's blood in their title and also a rock group with this name. However, this 
Table 1. Stem cells

\begin{tabular}{|c|c|c|c|}
\hline & Embryonic & Adult & Hematopoietic \\
\hline $\begin{array}{l}\text { Self-renewal } \\
\text { in vivo }\end{array}$ & Unlimited & Limited & Limited \\
\hline $\begin{array}{l}\text { Self-renewal } \\
\text { in vitro }\end{array}$ & Unlimited & Limited & Limited \\
\hline Potency & Totipotent & $\begin{array}{l}\text { Oligo- or uni- } \\
\text { potent }\end{array}$ & Multi-potent \\
\hline Self-renewal & Yes & Limited & Limited \\
\hline $\begin{array}{l}\text { Asymmetric cell } \\
\text { division }\end{array}$ & Yes & Yes & Yes \\
\hline $\begin{array}{l}\text { Symmetric cell } \\
\text { division }\end{array}$ & Unlimited & Limited & Limited \\
\hline Quiescence & No & Yes & Yes \\
\hline $\begin{array}{l}\text { Niche } \\
\text { dependence }\end{array}$ & No? & Yes & Yes \\
\hline
\end{tabular}

colorful practice has gone the way of the dodo bird replaced by more precise albeit less dramatic technologies such as the PCR, mass spectroscopy and whole-genome sequencing. And although umbilical cord blood is sometimes used as the source of a hematopoietic cell graft, it is this and not the normal blood cells which need a qualifier. We suggest using the term blood cell transplants rather than peripheral blood cell transplants. If nothing else, this will save a few trees.

Some colleagues may object to the term blood cell transplants as being too vague. For example, RBCs are blood cells but cannot engraft. However, it is safe to say that in the usual circumstances of transplants in humans we lack precise data of which of the cells in the blood collected by apheresis is responsible for recovery of BM function after high-dose therapy. This is even truer after less intensive pretransplant therapies. And we must consider the possibility that different cells in the graft are responsible for BM recovery in different persons. We are unlikely to trick ourselves into thinking that we know more than we do if we stick with blood cell transplants for now.

Myelo-ablative transplants. Another loser. Considerable data in experimental models, animals and humans clearly indicate that no dose of drugs or radiation irreversibly eradicates all BM cells capable of restoring BM function without also killing a person. This is easily understood as almost all modalities used to prepare people for a transplant kill hematopoietic cells (and other cells) by a stochastic process. This is especially so for ionizing radiations, but also applies to most radiomimetic drugs like the alkylating drugs. Death from non-BM effects occurs at a much lower dose of drugs or radiation than could possibly kill all BM cells capable of restoring hematopoiesis. This is not to say BM function will recover rapidly, it is simply that the term myeloablative is incorrect unless it is also person ablative. For example, persons accidentally exposed to TBI doses several times higher than a dose that would cause death within 30-60 days without supportive care, recover BM function after receiving antibiotics, antivirus drugs, molecularly cloned hematopoietic growth factors and RBC- and platelet transfusions. ${ }^{5}$ Using the term myelo-abaltive can result in serious mistakes in choosing therapy interventions. For example, one might feel obliged to do a transplant in someone who received a myelo-ablative dose of drugs or radiation even when autologous recovery is possible. Such a mistake could result in an iatrogenic death, for example, from GvHD. You may ask what to do with the CIBMTR classification of the intensity of pretransplant conditioning regimens. We suggest intensive or high-dose conditioning works just as well and is more accurate than myelo-ablative conditioning.

Engraftment. Widespread use of the term engraftment as synonymous with BM recovery is also a potentially serious inaccuracy. This was already a possible error when used after conventional high-dose pretransplant therapy but is especially so when pretransplant therapy is less intensive and/or when subsets of hematopoietic cells comprise the graft. We need to stick to what we know. After a transplant, what we typically quantify is recovery of BM function, not engraftment that can only be proved using genetic markers of the transplanted cells. Moreover, when genetic markers are used we need to specify which hematopoietic lineage(s) is engrafted: lymphocytes, myeloid cells, both or neither? And we also must distinguish between engraftment, which implies an active biological process and persistence of cells we infused without replication. Unless detailed genetic studies are done in a transplant recipient or cohort we are less likely to be wrong by describing what we measure, $B M$ recovery or recovery of BM function, rather than implying something we may not know with precision, engraftment.

Overall survival. We come next to OS. This seems a term created such that an abbreviation can be used, namely OS rather than for any scientific reason. Much like peripheral as in PBSC transplants this is an unnecessary modifier. Survival is survival when there is no preceding qualifier; overall adds nothing. We admit ' $S$ ' is not an attractive abbreviation for a chart but neither is 'OS'; both pale compared to elegant abbreviations such as RFS, DFS, EFS and the like. How about using survival on charts and graphs, which is only slightly longer than some of our favorite acronyms such as R-CHOP and MOPP/ABVD?

Autologous GvHD. Another peccadillo is autoGvHD. This term, which is closer to slang than jargon, immediately strikes one as an oxymoron. There is no convincing evidence of autoGvHD despite many publications. Although we do not dispute that some autotransplant recipients and recipients of genetically identical twin transplants develop clinical and laboratory features resembling GvHD, which occurs after an allotransplant, there is simply no proof these features are caused by the graft reacting against the host. Elsewhere we review these data critically. ${ }^{6}$ However, until there are convincing data regarding the pathophysiology of these clinical features, we are safer to limit our terminology to what we know. Affected persons can be described as having clinical features resembling allotransplant recipients with GvHD. (Several studies have shown there are no specific histological features of either autologous or allogenic GvHD).

There are many other examples of jargon in our field. We invite others to suggest terms needing revision, and expect this commentary will draw criticism and possibly rebuttals. However, it is important that we be precise in our terminology lest we think we understand more than we really do. We would be wise to follow the motto of the Royal Society in London: 'Nullius in verba' (Take nobody's word for it). Gentlemen (and ladies), start your engines of indignation; for every expert there is an equal and opposite expert. We welcome your comments; criticism less so.

\section{CONFLICT OF INTEREST}

RPG is a part-time employee of Celgene Corp., Summit, NJ, USA.

\section{ACKNOWLEDGEMENTS}

Professor John Goldman provided many insightful comments at the fireside. Dr Iman Saramipoor developed Table 1. RPG thanks the late Professor David W Golde for pointing out that Chilean sea bass sounds much better than Patagonian tooth fish (its real name). We acknowledge the support from the NIHR Biomedical Research Center funding scheme.

RP Gale ${ }^{1}$ and HM Lazarus ${ }^{2}$ ${ }^{1}$ Section of Haematology, Division of Experimental Medicine, Department of Medicine, Imperial College, London, UK and 
${ }^{2}$ Department of Medicine, Division of Hematology and Oncology, Case Western Reserve University, University Hospitals Case Medical Center, Cleveland, $\mathrm{OH}$, USA E-mail: robertpetergale@aol.com

\section{REFERENCES}

1 Gale RP, Eapen M, Logan B, Zhang MJ, Lazarus HM. Are there roles for observational database studies and structured quantification of expert opinion to answer therapy controversies in transplants? Bone Marrow Transplant 2009; 43: 435-446.
2 Enver T, Pera M, Peterson C, Andrews PW. Stem cell states, fates, and the rules of attraction. Cell Stem Cell 2009; 4: 387-397.

3 Peterburs MC. Bacterial Postmortem Examination of Heart's Blood, Spleen and Bile. Marquette University Press: Milwaukee, WI, USA, 1947.

4 Hamond CW, Tompkins M, Miller CP. Studies on susceptibility to infection following ionizing radiation. I. The time of onset and duration of the endogenous bacteremias in mice. J Exp Med 1954; 99: 405-410.

5 Baranov AE, Selidovkin GD, Butturini A, Gale RP. Hematopoietic recovery after 10-Gy acute total body radiation. Blood 1994; 83: 596-599.

6 Linhares Y, Lazarus H, Gale RP. What the heck is autologous GvHD?. Submitted. 